\title{
Unpolarized and polarized elementary kaon electroproduction cross sections measured at MAMI
}

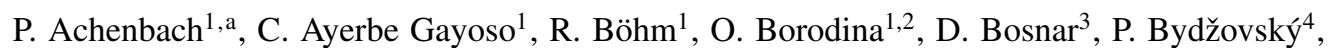
L. Debenjak ${ }^{5}$, M. Distler ${ }^{1}$, A. Esser ${ }^{1}$, I. Friščić ${ }^{3}$, Y. Fujii ${ }^{6}$, T. Gogami ${ }^{6}$, M. Gómez Rodríguez ${ }^{1}$, O. Hashimoto ${ }^{6, b}$, S. Hirose ${ }^{6}$, E. Kim ${ }^{2}$, A. Margaryan ${ }^{7}$, H. Merkel ${ }^{1}$, U. Müller ${ }^{1}$, S. Nagao ${ }^{6}$, S. N. Nakamura ${ }^{6}$, J. Pochodzalla ${ }^{1}$, C. Rappold ${ }^{2,8}$, J. Reinhold ${ }^{9}$, T. R. Saito ${ }^{1,2,8}$, A. Sanchez Lorente ${ }^{8}$,

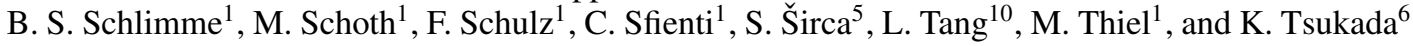
A1 Collaboration

1 Institut für Kernphysik, Johannes Gutenberg-Universität, Mainz, Germany

2 GSI Helmholtz Centre for Heavy Ion Research, Darmstadt, Germany

3 Department of Physics, University of Zagreb, Zagreb, Croatia

4 Nuclear Physics Institute, Řež near Prague, Czech Republic

5 University of Ljubljana and Institut "Jožef Stefan”, Ljubljana, Slovenia

6 Department of Physics, Tohoku University, Sendai, Japan

7 Yerevan Physics Institute, Yerevan, Armenia

8 Helmholtz Institute Mainz, Mainz, Germany

9 Department of Physics, Florida International University, Miami, FL USA

10 Department of Physics, Hampton University, Hampton, VA USA

\begin{abstract}
Present and future research into the electroproduction of kaons plays an important role at Mainz Microtron MAMI. With the Kaos spectrometer employed for kaon detection in the multi-spectrometer facility, cross section measurements of the exclusive $p\left(e, e^{\prime} K^{+}\right) \Lambda, \Sigma^{0}$ reactions at low momentum transfers have been performed. Isobar and Regge-plus-resonance models were compared with the data. These measurements have clearly discriminated between effective Lagrangian models for photo- and electroproduction of strangeness. New experiments with polarized beam at low four-momentum transfer are addressing the imaginary part of the longitudinal-transverse response in this process, that can be separated by flipping the beam electron helicity. These studies are important for the understanding of basic coupling constants in the isobar models and the electromagnetic form factors of the hadrons and their resonances involved in the process.
\end{abstract}

\section{Introduction}

To gain insight into the structure of hadrons it is important to study their excitation states. Resonances in the spectrum of nucleonic excitations reflect its internal structure. Information about these nucleon resonances are provided by experiments of photo- or electroproduction performed at electron accelerators worldwide, e.g. CEBAF at Jefferson Lab in the US, or MAMI and ELSA in Germany. At the Mainz Microtron MAMI research into electroproduction reactions that involve strangeness in the final state plays an important role. The presence of a strange quark pair $s \bar{s}$ in the reaction dynamics in addition to the up and down quarks can foster the understanding of the spectrum and dynamics of hadrons.

\footnotetext{
a e-mail: patrick@kph.uni-mainz.de

b deceased
}

This is an Open Access article distributed under the terms of the Creative Commons Attribution License 2.0, which permits unrestricted use, distribution, and reproduction in any medium, provided the original work is properly cited. 

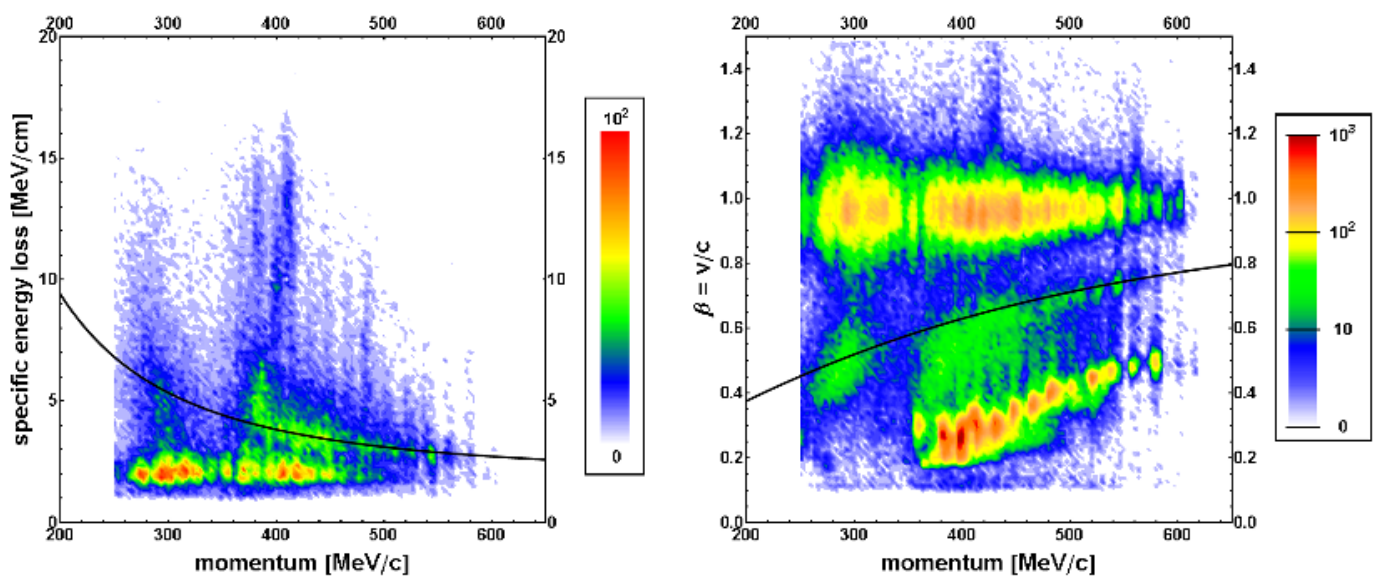

Figure 1. Identification of positively charged particles by their specific energy-loss (left) and their relative velocity (right) in the momentum range of the KAos spectrometer. The expectation for kaons is indicated by the solid lines. Pre-selection cuts have been applied to suppress the background of positrons and pions (found at $\mathrm{d} E / \mathrm{d} x \approx 2$ $3 \mathrm{MeV} / \mathrm{cm}$ and $\beta \approx 1$ ), and of protons (found at $\beta \approx 0.2-0.4$ ).

In the elementary kaon electroproduction reaction the virtual photon cross section for a longitudinal polarized electron beam described by helicity eigenstates $h= \pm 1$, unpolarized target and unobserved spin degree-of-freedom in the final state can be cast into the form:

$$
d \sigma_{o} / d \Omega_{K}^{c . m .}=\sigma_{T}+\epsilon \sigma_{L}+\epsilon \sigma_{T T} \cos 2 \phi+\sqrt{2 \epsilon(1+\epsilon)} \sigma_{L T} \cos \phi+h \sqrt{2 \epsilon(1-\epsilon)} \sigma_{L T^{\prime}} \sin \phi,
$$

where $\sigma_{i}$ are structure functions with $i=T, L$, and $T T$ representing the transverse, longitudinal, and transverse-transverse term, while $L T$ and $L T^{\prime}$ describe transverse-longitudinal interferences. The kaon angular differential cross section is determined in the hadronic center-of-mass system (c.m.) of virtual photon and target.

Unpolarized kaon photo- and electroproduction measurements with high statistics have been performed at Jefferson Lab [1-5], where also a first polarized measurement was done with the CLAS spectrometer [6]. This spectrometer could access a kinematic region of four-momentum transfers to the proton $Q^{2}>0.5(\mathrm{GeV} / c)^{2}$ which is complemented by the measurements at MAMI.

\section{Experimental setup and data analysis}

The Mainz Microtron MAMI provides a continuous-wave electron beam with energies up to $1.6 \mathrm{GeV}$ with a very small halo and good energy definition. Kaon electroproduction experiments at MAMI were performed at the multi-spectrometer set-up of the A1 Collaboration [7] with a beam of $1.507 \mathrm{GeV}$ energy delivered on a liquid hydrogen target with a length of $5 \mathrm{~cm}$. For the detection of the electron, high-resolution Spectrometer B with a solid angle acceptance of $5.6 \mathrm{msr}$ and a momentum acceptance of $15 \%$ was used. Kaons were detected in the KaOs spectrometer positioned at an angle of around $38^{\circ}$, whose performance has been discussed in detail elsewhere [8].

With this set-up unpolarized cross section measurements of the exclusive $p\left(e, e^{\prime} K^{+}\right) \Lambda, \Sigma^{0}$ electroproduction reactions were performed in the kinematic region of low four-momentum transfers [9] [10]. In these proceedings the first measurement of a polarization observable in kaon electroproduction at MAMI is described. This experiment used Spectrometer B at an in-plane angle of only $14^{\circ}$ and an out-of-plane angle of $10^{\circ}$, leading to angles $\phi \approx 40^{\circ}$ between the electron-scattering and hadronproduction plane, and used a spin-polarized electron beam in which the electron helicity state $h$ was flipped once per second.

The beam polarization was measured with a Møller polarimeter regularly and the average beam polarization was $P_{\text {beam }}=87 \%$. The polarimeter setup utilizes an array of lead glass detectors and a 


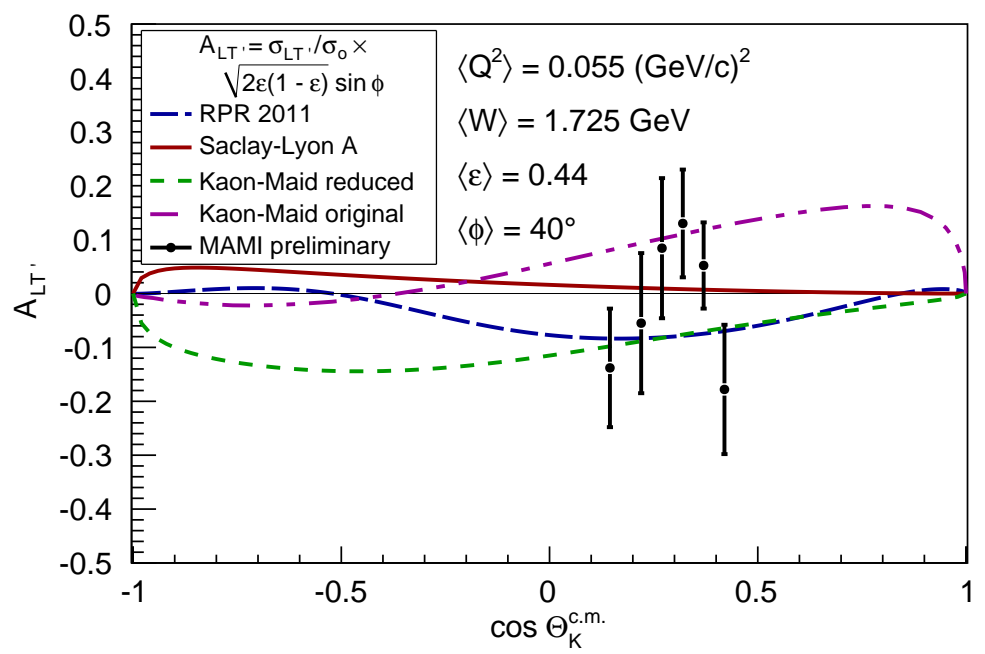

Figure 2. Model predictions and preliminary data points from MAMI for the beam helicity asymmetry in the exclusive $K^{+} \Lambda$ electroproduction reaction. Statistical errors are shown with the preliminary data. "RPR 2011" is a Regge-plus-resonance model developed by the Ghent group [11, 12]; "Kaon-Maid original" refers to the original Kaon-Maid model [13]; in "Kaon-Maid reduced" the longitudinal couplings to the nucleon resonances were removed; and "Saclay-Lyon A" is yet another isobar model using a different set of nucleon resonances [14].

dipole magnetic spectrometer for a two-arm coincidence measurement of the scattered electron with recoiling target electron from a magnetized iron target foil.

The data analysis used the same technique that was reported earlier [9]. For the polarized electroproduction measurements the Kaos spectrometer was modified to improve the particle identification power: (i) the distance between scintillator walls was chosen to satisfy $\Delta L>1 \mathrm{~m}$; (ii) a new aerogel Cherenkov detector was installed for pion and positron suppression; (iii) a new FPGA-based trigger was implemented; and (iv) a new tracking code was developed to handle higher beam currents. Kaon candidates were selected by measurements of energy-loss and flight-time in the Kaos spectrometer, see Fig. 1 , and the $\left(e^{\prime} K^{+}\right)$reaction was identified by the coincidence time measurement between the two spectrometers with a resolution of $\sim 1 \mathrm{~ns}$. The spectroscopy of kaons and electrons allowed the reconstruction of the undetected hyperon, and consequently, the separation of the $K^{+} \Lambda$ and $K^{+} \Sigma^{0}$ reaction channels. After background subtraction a total yield of $N_{K^{+} \Lambda} \sim 1500$ pairs in the $\Lambda$ hyperon channel were identified. Fig. 2 shows the preliminary asymmetry $A_{\text {exp }} \cdot P_{\text {beam }}=\left(N^{+}-N^{-}\right) /\left(N^{+}+N^{-}\right)$ determined from positive and negative beam helicity states.

\section{Discussion of cross sections and beam helicity asymmetry}

In these experiments the proton was probed in its third resonance region at energies of $W=1.6-$ $1.7 \mathrm{GeV}$ and the unpolarized cross sections were taken at low four-momentum transfers $Q^{2}=0.03-$ $0.055(\mathrm{GeV} / c)^{2}$. With $Q^{2}$ being a measure of the photon's virtuality, these experiments used almost real photons, although with a relatively large degree of transverse linear polarization $\epsilon=0.4-0.5$. Longitudinal and longitudinal-transverse interference terms are strongly suppressed in the total cross section by the small value of $Q^{2} / \omega^{2}$ with $\omega \sim 1-1.2 \mathrm{GeV}$ as the photon's energy. With the virtual photons partially linearly polarized, the electroproduction cross section at low $Q^{2}$ complements the unpolarized photoproduction cross section. Those phenomenological models developed for kaon photoproduction in which the contribution of longitudinal structure functions in the models is small are in agreement with the data. The first experiments showed that the $Q^{2}$ dependence of the cross section is flat. However, the separation of structure functions in this region remained desirable. In the latest experiment at $Q^{2} \sim 0.055(\mathrm{GeV} / c)^{2}$ the polarized structure function in the differential cross section $d \sigma / d \Omega_{K}^{c . m}$. could 
be accessed through the helicity asymmetry:

$$
A_{L T^{\prime}}=\frac{d \sigma^{+} / d \Omega_{K}^{c . m .}-d \sigma^{-} / d \Omega_{K}^{c . m .}}{d \sigma^{+} / d \Omega_{K}^{c . m .}+d \sigma^{-} / d \Omega_{K}^{c . m .}}=\sqrt{2 \epsilon(1-\epsilon)} \sigma_{L T^{\prime}} / \sigma_{o} \sin \phi .
$$

This structure function can be constructed from the imaginary part of the longitudinal-transverse response in this process and is sensitive to the interferences between resonant and non-resonant amplitudes in the probed energy region around $1.725 \mathrm{GeV}$.

Theoretically the elementary process for $K^{+} \Lambda$ production on the proton can be described by utilizing isobar models. The background terms of such models consist of $s^{-}, t-$, and $u$ - channel Born terms and kaon and hyperon resonances. Model predictions for the unpolarized and polarized structure function are available from variants of the Kaon-Maid model [13] and from the Saclay-Lyon [14] and Regge-plus-resonance [11, 12] models, see also Fig. 2. A main topic of discussions is the choice of nucleon, kaon, and hyperon resonances in the models. The nucleon resonances $N(M) L_{2 I ; 2 J}$ of mass $M$ are characterized according to their orbital angular momentum $L$ of the partial wave, their isospin $I$, and their spin $J$. In the Kaon-Maid model the cross section at $W=1.725 \mathrm{GeV}$ is dominated by the $s$-channel resonances $N(1720) P_{13}$ and $N(1650) S_{11}$. These resonances together with non-resonant contributions from Born and $K^{*}(890)$ exchange terms give the largest strength. In the Saclay-Lyon model the contributions from $N(1680) F_{15}$ and $K_{1}(1270)$ plus $K^{*}(890)$ exchanges appear to be important. Two hyperon resonances reduce the divergence of the Born terms at high energies. In the Regge-plus-resonance model the background terms are provided by a Regge model in which two kaon trajectories from the $K^{+}(494)$ and $K^{*+}(892)$ are exchanged. Several nucleon resonances are included in the resonance part of the amplitude.

\section{Acknowledgments}

Work supported in part by the Federal State of Rhineland-Palatinate and by the Deutsche Forschungsgemeinschaft (DFG) with the Collaborative Research Center 1044, by the Research Center "Elementary Forces and Mathematical Foundations", and by the Carl Zeiss Foundation. We also acknowledge the support by the Research Infrastructure Integrating Activity "Study of Strongly Interacting Matter" HadronPhysics 2 under the 7th Framework Programme of EU and by the Grant Agency of the Czech Republic, grant No. 203/12/2116. The Japanese group of the Collaboration acknowledges the support by Strategic Young Researcher Overseas Visits Program for Accelerating Brain Circulation (R2201). This work is also supported in part by the Core-to-Core program of Japan Society for Promotion of

Science (200102).

\section{References}

1. R.M. Mohring et al. (E93-018 Collaboration), Phys. Rev. C 67, 055205 (2003)

2. R. Bradford et al. (CLAS Collaboration), Phys. Rev. C 73, 035202 (2006)

3. P. Ambrozewicz et al. (CLAS Collaboration), Phys. Rev. C 75, 045203 (2007)

4. M. Coman et al. (Hall A Collaboration), Phys. Rev. C 81, 052201 (2010)

5. M.E. McCracken et al. (CLAS Collaboration), Phys. Rev. C 81, 025201 (2010)

6. R. Nasseripour et al. (CLAS Collaboration), Phys. Rev. C 77, 065208 (2008)

7. K.I. Blomqvist et al. (A1 Collaboration), Nucl. Instr. and Meth. Phys. Res. A 403, 263 (1998)

8. P. Achenbach (A1 Collaboration), Eur. Phys. J. Special Topics 198, 307 (2011)

9. P. Achenbach et al. (A1 Collaboration), Eur. Phys. J. A 48, 14 (2012)

10. P. Achenbach et al. (A1 Collaboration), Nucl. Phys. A 881, 187 (2012)

11. T. Corthals, D.G. Ireland, T. Van Cauteren, J. Ryckebusch, Phys. Rev. C 75, 045204 (2007)

12. L. De Cruz, T. Vrancx, P. Vancraeyveld, J. Ryckebusch, Phys. Rev. Lett. 108, 182002 (2012)

13. T. Mart, C. Bennhold, Phys. Rev. C 61, 012201 (2000)

14. J.C. David, C. Fayard, G.H. Lamot, B. Saghai, Phys. Rev. C 53, 2613 (1996) 